\title{
Smell and touch in the Virtual Jumpcube
}

\author{
Horst Eidenberger ${ }^{1}[$
}

Received: 17 August 2017 / Accepted: 22 August 2018 / Published online: 1 September 2018

(c) The Author(s) 2018

\begin{abstract}
The Virtual Jumpcube is a virtual reality setup from 2015 that allows for jumping and flying in audiovisual virtual environments. Recently, we have included several haptic and olfactory stimuli that should further increase the degree of immersion in the experienced virtuality. These additional media channels were tested by the participants of several events and the feedback of 196 jumpers was gathered in a questionnaire. In this paper, we describe the stimulation hardware and software as well as the performed experiment and we present the major findings of the evaluation. It shows that if employed correctly, haptic and olfactory stimuli can enhance immersion and user experience significantly. Major success factors appear to be the amplitude and frequency of stimulation as well as the temporal synchronization with the other media channels, in particular the visual stimuli.
\end{abstract}

Keywords Virtual reality $\cdot$ Olfactory stimuli $\cdot$ Haptic stimuli $\cdot$ Force simulation $\cdot$ User-based evaluation

\section{Introduction}

\subsection{Goals and motivation}

Two major design goals of virtual reality applications are the maximization of immersion and the avoidance of nausea (or motion sickness) in the users [3, 19]. The immersion in the virtual world can be seen as the degree to which we forget the outside world during the experience. In entertainment applications, the maximization of excitement is one further goal of virtual reality as a media system $[6,14]$. Often, the same information could be conveyed over other media channels as well though virtual reality allows a more lively information process with potentially higher impact in the receivers. It appears to be obvious that immersion, nausea and excitement are linked phenomena: nausea destroys immersion and excitement, immersion increases excitement and vice versa. Consequently, the optimization of a virtual reality application is a multidimensional problem that requires careful design of the solution.

Communicated by R. Steinmetz.

Horst Eidenberger

horst.eidenberger@tuwien.ac.at

1 TU Wien, Interactive Media Systems Group, Favoritenstrasse 9/19306, 1040 Vienna, Austria
In this paper, we describe how additional media streams have been employed to maximize immersion, minimize nausea and maximize excitement in one particular virtual reality project, the Virtual Jumpcube $[4,20]$. The media under consideration are olfactory and tactile ${ }^{1}$ stimuli. The scientific contribution of the paper is the description of the stimulation hardware and software system for these non-standard media, extended testing with almost 200 subjects and a detailed analysis of the results. The underlying assumption is that smell and touch are efficient means to maximize immersion and excitement while minimizing motion sickness. We perceive virtual reality as a multimedia application where audiovisual channels are augmented by the olfactory and tactile stimulation streams and assume that-like for audiovisual media streams-precise synchronization is the key to the efficient usage of these media types [6,14]. Virtual reality in general and the Virtual Jumpcube setup in particular appear highly suitable for the investigation of smell and touch since these environments are rather static in comparison to, for example, digital video that can be consumed almost anytime anywhere nowadays.

The present investigation is a first step in our endeavor to integrate non-audiovisual stimulations into hybrid virtual reality experiences. It is afflicted with several limitations

\footnotetext{
"We use "haptic" and "tactile" as almost synonymous terms in this paper, referring to local stimulation as "haptic" (e.g., force simulation by a rope dragging at the hips) and to areal stimulation as "tactile" (e.g., wind).
} 
Fig. 1 In the Virtual Jumpcube, jumpers enter at the back, jump towards the front and leave the cube at the front (a). During flight, jumpers can move leftright and up-down (b). The levers and discs in $\mathbf{b}$ on the left are explained in Sect. 2
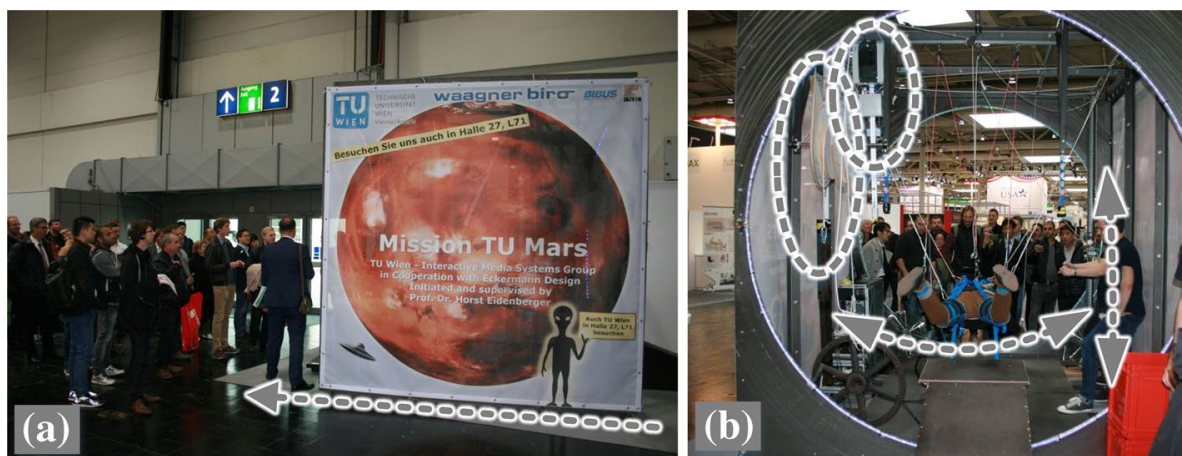

that are in their majority caused by the business model of this research (see Sect. 3.2 for details). First, the subjects that use the Virtual Jumpcube are guests of some event (e.g., a congress) that pays for the participation of the cube. Hence, we cannot switch off certain stimuli for some of the participants thus creating a comparison group. Under these circumstances, the questionnaires handed out to the subjects have to be relatively short and the usage of technology has to be conservative: every component employed has to be well tested. Furthermore, the present setup was of course not made for the evaluation of nausea but the opposite: we endeavored to include as many promising solutions science offers for the avoidance of motion sickness as possible. Likewise, all audiovisual contents presented in the Virtual Jumpcube are designed for maximal excitement. Therefore, the level for the new olfactory-tactile stimuli is already rather high. There are, however, also mitigating factors and benefits from the application scenario. For example, since a considerable part of the population suffer from partial or total anosmia (up to $20 \%$ according to [12]) these subjects can be employed as a comparison group for olfactory stimulation. Moreover, the paramount advantage of this form of testing is that the results reflect the true situation of virtual reality and its defining parameters immersion, nausea and excitement: every subject used the Virtual Jumpcube out of her/his own curiosity in a real-world environment, thus guaranteeing the practical representativeness of the results.

The Virtual Jumpcube setup was first described in [4]. In brief, it is a complex system of hardware components that allows one subject to perform a free jump, flight and landing sequence in the real world while experiencing a virtual world through virtual reality gear. Figure 1 shows the cube in action at the Hannover industrial fair. The development of the majority of the hardware components was necessary for making sure that the unique aspect of the cube-free full-force jumping forward-can be performed without the risk of injury. This aspect also constitutes the unique selling factor of the setup and was approved by almost two thousand jumpers since April 2015: jumping is fun for the acting person but as well for the watching audience, an aspect that made this research prototype attractive for the presentation at tech-shows and related events.

\subsection{Overview}

Below, we describe how the Virtual Jumpcube was extended by hardware and software for the presentation of olfactory and tactile stimuli (Sect. 2). Then, in Sect. 3, we specify the experiment that was performed on the setup, explaining all relevant parameters and constraints. At the heart of Sect. 3 are eleven hypotheses that are evaluated on the basis of the results presented in Sect. 4. Next to hypothesis testing, we also perform a general evaluation of the results based on descriptive statistics and conclude on the results in the last section of the paper where we also give an outlook on future scientific investigations in the context of the Virtual Jumpcube project. But first we sketch the present situation in virtual reality smell and touch research in the last section of the introduction.

\subsection{Related literature}

The Virtual Jumpcube is an example for what is sometimes called the interface challenge in virtual reality research [3, 19]. Many more creative, complex, intriguing hardware prototypes have been built in the past decades. Recently, two further flying (though not jumping) setups have gained attention in the region of the author: Birdly [17] is a wellknown system for bird-like flight through virtual worlds. In addition to audiovisual media streams it contains two major haptic channels: first, the user has to flap his/her wings to build up speed, and second, wind is blown into the face of the user. Another flight application is Icaros [10]. Icaros is - in comparison to Birdly and the Virtual Jumpcube - a rather simple hardware setup that allows the user to balance herself and thus experience a flight-like virtual environment. Birdly and Icaros are two beautiful examples for virtual reality flight gear. We assume that further sophisticated flight applications do exist though, to the knowledge of the author, none of these systems would 
allow the user to perform a free jump, which is a unique experience in itself.

Next to flight applications, a considerable number of walking, swimming and other movement applications were proposed in recent years. We would like to point out [5] for virtual swimming, [13] for gravity reduced stepping and [1] for walking, running and vertical jumping in static environments. Since movement is essential in these setups they must be considered as integrating to a certain degree haptic aspects. Yet to the author's knowledge they do not explicitly provide tactile stimuli of the skin nor olfactory stimulation. Smells in particular have seen only little application in virtual reality recently. Since the spectacular failures of Smell-o-Vision and Aroma-Rama in the 1960s [3], only little has been done to augment multimedia and virtual reality systems by such stimuli. One notable exception are the works by the group of Alan Chalmers at the University of Warwick $[2,16]$. In the area of touch, the situation is significantly more diverse [11]. Numerous systems have been proposed for skin stimulation through gloves, vibration motors, heat and cold. Setups have been built for force simulation and feedback (e.g., [13]) as well as for the encoding of cognitive information in rhythm patterns (for example, the well-known HTC Vive archery game).

Below, where necessary we refer to (recent) discoveries in neuroscience and psychology on the operating mode of our sensor systems for olfactory and tactile perception. This knowledge sources mostly from [12] and-where psychophysical aspects are concerned-from [8]. Knowledge on touch perception, in particular large-field stimulation of the skin, were taken from $[7,15]$. For example, we employ the fascinating findings of neuroscience for the design of appropriate olfactory stimulation streams (see Sect. 2.3) that endeavor to contribute to the overall goal of maximal immersion as efficiently as possible.

\section{Setup}

This section summarizes the hardware employed in the described experiment. Where necessary, we refer to earlier descriptions of the Virtual Jumpcube. In particular, we describe the smell system and the three tactile components used for skin stimulation.

\subsection{The Virtual Jumpcube}

The Virtual Jumpcube [20] was built as a demonstration object for the potentials of virtual reality in the context of the 200th anniversary of TU Wien in the winter 2014/2015. In [4], we give an introduction of the original system comprising of the frame, suspension system and virtual reality gear and software. Since then, the system has been extended by modules for olfactory and haptic stimulation as well as various contents and scenarios (originally, skydiving, now as well diving, space travel and air racing). Still, the fundamental goal remains the same: it is providing an infrastructure for free jumping and flying in virtual environments. The suspension is based on a system of ropes, pulleys and counterweights balanced by eccentric discs that map the nonlinear force curve of the jump onto the static counterweights.

Free jumping while being locked into a virtual environment constitutes a loss of control situation that should influence the perception of the virtual content-hopefully making it more exciting. Major aspects of the installation are the usage of rich media streams, demonstratively visible safety and portability/modularity of the entire setup. Rich media usage means that while the audio tracks of some content contain as well sound effects, background music andwhere necessary - the voice of an instructor, the visual layer is always composed of as complex as possible $3 \mathrm{D}$ models, special effects and a layer for game play thus combating the fundamental disadvantage of virtual reality (in comparison to 3D movies-the other 3D media type familiar to most users) that the scene has to be rendered ad hoc.

Since the frame of the cube is relatively big $(320 \mathrm{~cm}$ in each dimension), it can accommodate the hardware modules for new stimuli comfortably. We therefore found it a suitable platform for evaluating the above-mentioned ideas that smell and touch might increase immersion and excitement while reducing the risk of nausea. The only difficulty we encountered was the fact that we could not find any readymades for the stimulations we had in mind. Hence, we had to construct our own prototypes. They are described in the Sects. 2.3 and 2.4. Before that the next section introduces the general architecture of the Jumpcube setup.

\subsection{System architecture}

The Jumpcube system consists of input, processing and output components. Figure 2 illustrates the interaction of the components. The central element is the application controller which manages input and output components as well as the virtual reality applications. The application controller exists in two implementations: one is based on the Unity game engine, the other on Unreal. Both consume content in the form of 3D models from the attached content database. Sensory input is acquired from connected sensors, in particular the hand-helds of the employed HTC Vive headmounted display and a self-developed acceleration-based motion sensor for flying applications. Audiovisual output is propagated from the application controller to the headmounted display and connected headphones.

All other output components-those that provide the accompanying touch and smell stimuli-comprise of a 
Fig. 2 At the core of the Virtual Jumpcube are two application controllers, one based on the game engine Unity, the other on Unreal. Apps such as a virtual skydive are managed by the app controllers. They receive input from sensors and the content database. Audiovisual output is provided by standard VR gear. Smell and touch output are created by production units (e.g., fans, heater elements) managed by self-developed controller units. All controllers use the same protocol propagated over a LAN through a socket interface. A browser-based console allows overriding apps as well as calibrating and configuring controller units

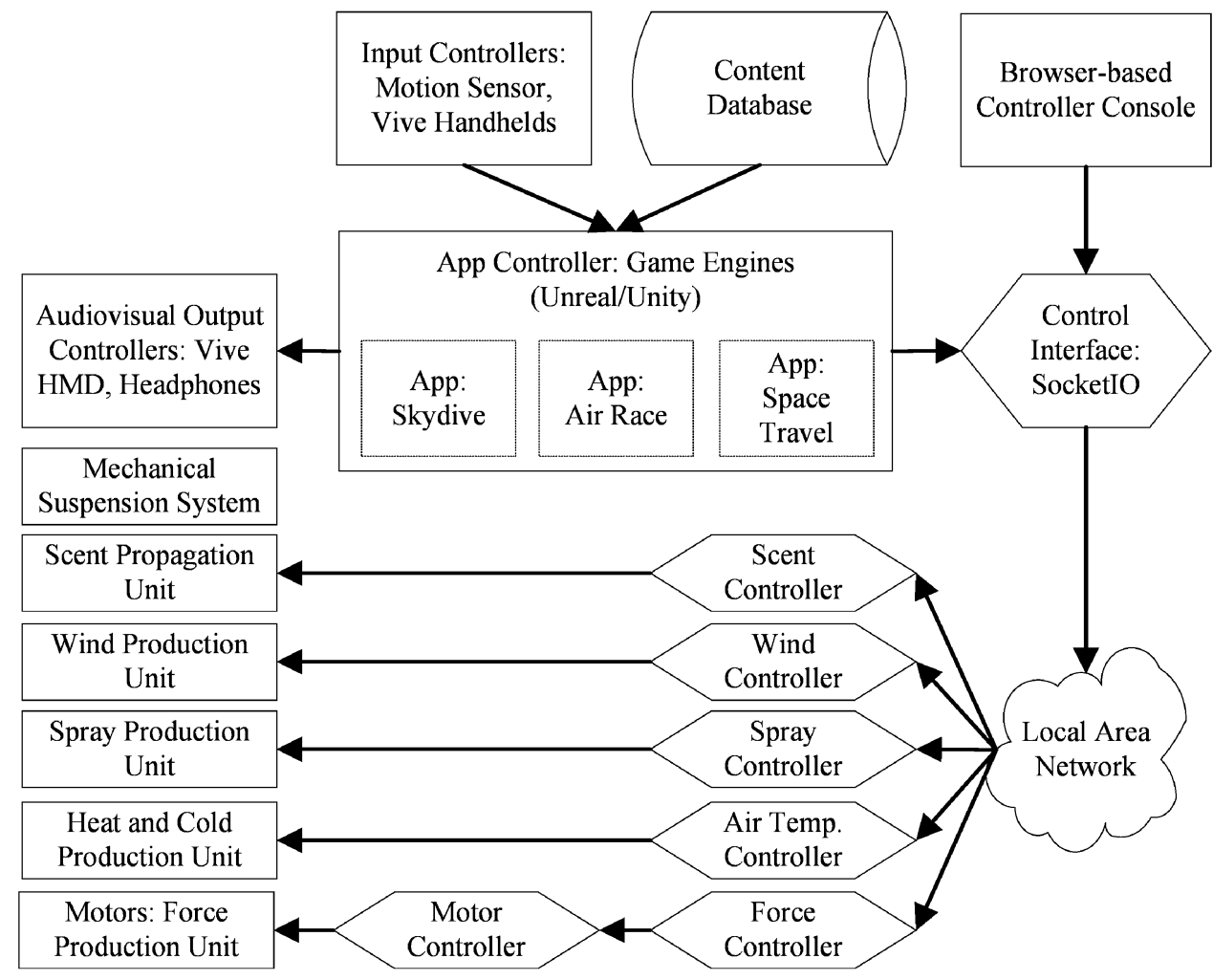

production unit and a managing controller. Production units include fans, heaters, a cooling device, a smell propagation system and a motor for force simulation. All managing controllers were developed during the project. Each consists of an embedded processor (mostly, Raspberry Pi and Arduino boards) and individual control software with a common software interface. The common interface is based on a selfdefined control protocol. The protocol defines a simple proprietary JSON dialect for controlling production units (start/ stop) and the setting of parameters (attributes and ranges of values). Messages are propagated over a local area network through a socket interface. Furthermore, for calibration and configuration purposes, we provide a browser-based console.

The system architecture includes one notable exception. The suspension system is independent from all other components and purely mechanical. For users with a weight of ca. $30-150 \mathrm{~kg}$ and a height of $130-196 \mathrm{~cm}$, it requires no configuration nor calibration. Being made from high-quality sailing and mountaineering equipment, it provides suspension without any notable time delay and the decoupling from the rest of the system guarantees the meeting of legal safety requirements.

The development of the system architecture progressed in an evolutionary bottom-up design process in the following steps: first, we defined the component architecture of dedicated controllers for specific stimulus production units. In the second step, we formulated a TCP-based protocol that is sufficiently general for managing a variety of different production units. This protocol was implemented in a software library that is accessible from both considered game engines and provides a web-based console interface. Based on production, controller and network hardware the two application controllers were implemented next. In this process, particular emphasis was given to the important task of 3D content management. Finally, the entire system was iteratively refined during the development of the three first apps for skydiving, space travel (both unity-based) and air racing (unreal-based).

The present system architecture proofed successful in its ability to integrate new stimulation components. Controllers make use of the implemented control protocol. The present software library can be accessed from most state-of-the-art embedded processors and boards. Since controllers encapsulate the production units, almost any thinkable stimulation hardware can easily be integrated into the environment by just plugging the controller into the local network and registering it via the console. The next two sections describe the most important of these components for smell and touch stimulation in the Virtual Jumpcube.

\subsection{Olfactory stimuli: the Vragrancer system}

Figure 3 depicts the various elements of the Vragrancer, the smell provider in the Virtual Jumpcube. It is based on a product named smell controller developed by a German company in the 1990s for usage in smell cinemas. 

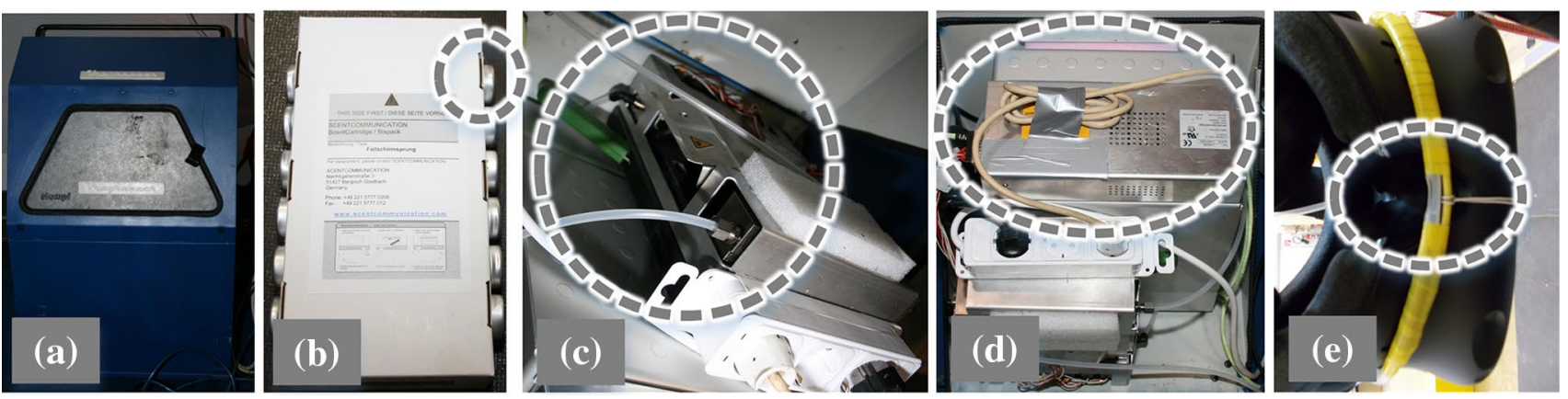

Fig. 3 The Vragrancer system is integrated in a mobile case (a). A cartridge consists of six smells that can be selected from a list of 5000 (b). The cartridge is loaded into the smell controller (c), which is controlled by a Raspberry Pi (d). Through a tube, smells arrive at the nose in an open circular tube that is connected via a $\mathrm{T}$-shaped push-in connector at the top of the head-mounted display (e)
Yet it was never launched and we only found it by coincidence. The smell controller is loaded with cartridges of six smells, each encapsulated in a sealed vial where the liquid smell is held by crystals. On request, the developing company (now operating in the marketing industry) provided us with a stock of smell cartridges for three virtual experiences: skydiving, space travel and air racing. Smells include combustion engine, smoke, coffee, and flowers. All smells are synthetic, sometimes not particularly pleasant yet thematically recognizable. Most importantly, they are approved as harmless by European authorities.

For usage of the smell controller in our system, we built a controller, based on a Raspberry Pi microprocessor and an Arduino for valve control. Communication between game engine and controller is performed over a TCP socket based on the before-mentioned JSON protocol. Smells are propagated by sending compressed air through valves and then over pipes (bundled with the cables of the head-mounted display) to the nose where an opening in the pipe delivers the smell-enriched air directly under the nose. The entire system with a pressure of just one Bar and a pipe length of approximately five meters has a surprisingly low latency of about $100 \mathrm{~ms}$. Embedded in a compact, mobile case the Vragrancer system can be configured with arbitrary smell combinations and can be employed in arbitrary (semi-)static virtual reality applications.

Essential to the Vragrancer is the usage pattern. From the above-mentioned failure of Aroma-Rama and related projects-where smells were used constantly as themes to encode situations and actors-we derived the conclusion that smell is a stimulus essentially different from the audiovisual ones. While we are used to holistic permanent application of the latter stimulus type, a constant stream of smells might overload the consumer. Rather, smells should be used selectively and only in situations where they are well-motivated and recognizable. For such application cartridges of six smells each appeared sufficient for the five minutes experiences of the Jumpcube.

\subsection{Haptic stimuli}

Three haptic modules were employed in the present evaluation: the jump suspension system, the weather system and a force simulation system. They are described below. A fourth haptic component available in the Jumpcube, the vibration system that effects on hands and hips, is not described as it was not part of the evaluation. Such vibration patterns are, for example, employed for making explosions more realistic and for simulating water resistance in a reef diving application.

\subsubsection{Jump suspension system}

Figure 4 illustrates the effect of the jump suspension system. It was described in detail in [4] and the central idea of the eccentric discs (a form of an analog computer) was sketched above. We consider the suspension system a haptic module because it enables the jumping which is a haptic experience. Furthermore, it also includes a winch for raising the flying subject (for example, when the parachute opens) that is also employed for the simulation of turbulences during the flight. A last component of the suspension system is the flight suit that contributes significantly to the tactile perception of the Virtual Jumpcube. Several of the investigated questions (listed in Sect. 3.1) are targeted at the suspension system.

\subsubsection{Weather system: wind, spray, heat, cold}

The weather system (see Fig. 5) of the Jumpcube is mostly integrated into the floor of the cube. It consists of wind engines at various positions of the cube, heat sources, spray and a source of cold air. All components are controlled by 

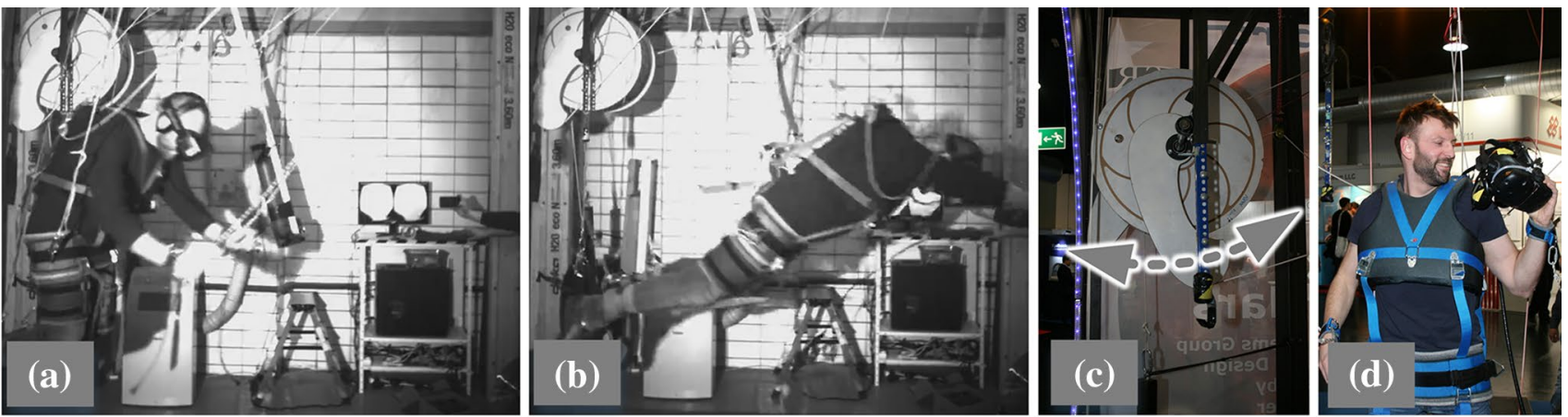

Fig. 4 The suspension system has to eliminate forces into the forward-down direction $(\mathbf{a}, \mathbf{b})$. Forces are balanced by rope-connected eccentric discs and counterweights (c). The tailor-made jump suit also contributes to the force elimination (d)
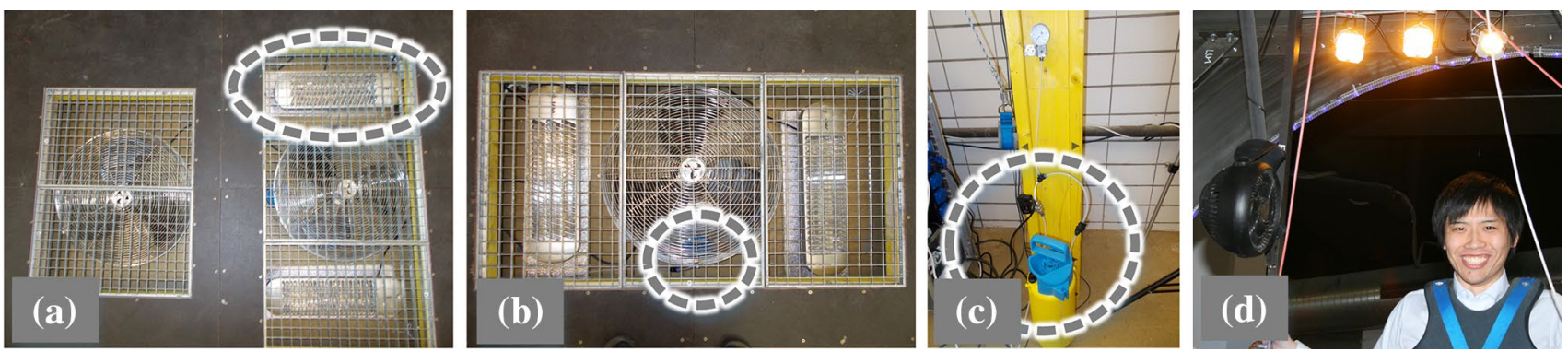

Fig. 5 Currently, the weather system consists of elements for wind $(\mathbf{a}, \mathbf{b}, \mathbf{d})$, heat (top right element in $\mathbf{a}$, side elements in $\mathbf{b}$ ), and spray (center bottom element in $\mathbf{b}, \mathbf{c})$. An additional component for cold air is under development

the application through the game engine and the attached controllers. Parameters are rotation speed, temperature and the amount of spray injected in the wind stream. The same type of controller and communication system as for the Vragrancer is employed for the weather system and again, latencies are minimal. In the present evaluation, only the wind and spray subsystems were employed. Spray is, for example, used for making clouds more realistic and when touching a water surface during the air race. Hot and cold air-for example, employed to express the coldness of space and the heat of the sun-will be made the subject of a future evaluation.

\subsubsection{Force simulation}

The third haptic component considered in the present evaluation is a system for the simulation of centrifugal forces. It consists of a motor, an electric clutch and a system of ropes that pulls at the hips of the flying subject. Triggered by the application, changes of flight direction are expressed visually (and sometimes, audibly) and supported by a sharp pull at the hips plus slow dragging for the time of the looping. The clutch is just a safety measure: when the end of the control rope is reached the clutch opens, thus decoupling the motor. Then, through gravitation the subject and the suspension system return to a stable centralized position.

The force simulation system is the only Jumpcube component that employs two controllers. The first one serves as interface to the control protocol, decoding and propagating control messages from the application controller or the console. The second controller, provided by the manufacturer of the motor performs the motor management in standardized CAN fashion. As can be seen from Fig. 6, the force simulation system is mounted at one of the levers used in the suspension system for calibration. Despite that it is an independent system that could also be employed outside of the Jumpcube.

\section{Experiment}

After stating the general idea of the research in the introduction and sketching the employed hardware in the last section, the three parts of this section describe the experimental design: first, the hypotheses into which the idea was operationalized, then the test environment, and finally, the participants of the experiment. 

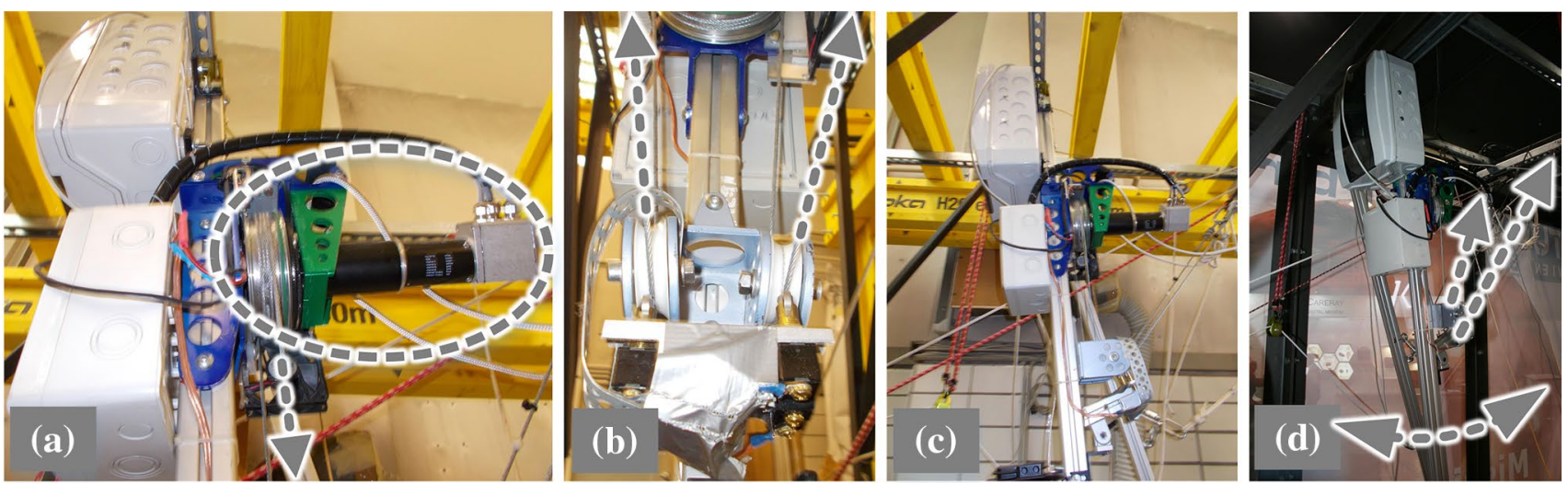

Fig. 6 The force simulation system consists of a quick-response motor that is connected to ropes via a magnetic clutch (a). The ropes drag at the hip belt of the jumper, thus creating the impression of cen- trifugal forces $(\mathbf{b}, \mathbf{c})$. In the Jumpcube, the force simulation system is attached to one of the levers of the suspension system (d)

Table 1 Hypotheses on the influence of smell and touch on humans in the Virtual Jumpcube

\begin{tabular}{ll}
\hline No. & Hypothesis \\
\hline H.O1 & Subjects that perceive olfactory stimuli as synchronous with the virtual reality content reach a rather higher level of immersion \\
H.O2 & Olfactory stimuli with adequate intensity are rather perceived as pleasant independent of their actual smell \\
H.O3 & The perception of smells reduces the risk of nausea \\
H.O4 & People who can perceive smells find the experience more often very exciting \\
H.H1 & Jumping is as exciting for elderly subjects as for younger subjects \\
H.H2 & The perception of centrifugal forces will rather reduce the risk of nausea \\
H.H3 & Centrifugal forces in curves are experienced as realistic no matter if the content is realistic or not \\
H.H4 & Spray makes clouds rather more exciting \\
H.G1 & Subjects that perceive olfactory and haptic stimuli more often reach a high level of immersion \\
H.G2 & Subjects that reach a higher level of immersion find the experience more exciting \\
H.G3 & Subjects with virtual reality experience react stronger on olfactory and haptic stimuli than subjects without experience
\end{tabular}

\subsection{Hypotheses}

Table 1 lists the hypotheses that were selected from a pool of ideas for the evaluation of the impact of smell and touch on immersion, excitement and motion sickness in virtual reality applications. Four hypotheses refer to olfactory stimuli (H.O1-4), four to haptic stimuli (H.H1-4) and three concern both types of stimuli. Of course, with the data available other hypotheses could be formulated and tested. We chose this set out of representativeness, clarity and compactness considerations. For further analysis, the curious reader may download the raw data of the evaluation from [9].

The underlying assumption of the hypotheses is that smell and touch improve immersion and excitement while reducing the effect of nausea. The majority of the statements expresses this belief. Some additional hypotheses (e.g. H.H1) were included for clarifying the effect of certain characteristics of the test subjects on the generality of the results. Please note that the hypotheses are formulated independent of the employed audiovisual content. In the next section, we will outline that three different types of audiovisual content were employed in the present experiment. Since our interest is to derive general conclusions on the usage of touch and smell stimuli, these content variants employ exactly the same types of olfactory and haptic stimuli with exactly the same parametrization (though at different locations of the content, of course). In consequence, the results should as far as possible be independent of the employed content semantics.

Below, in addition to hypothesis testing, we provide descriptive statistics of the evaluation results-one the one hand, for broadening the picture drawn by the evaluation, and on the other hand, for deepening the discussion of the results of hypothesis testing.

\subsection{Experiment design}

This section sketches the test environment briefly, gives an idea of the 3D contents used in the evaluation, and finally, 

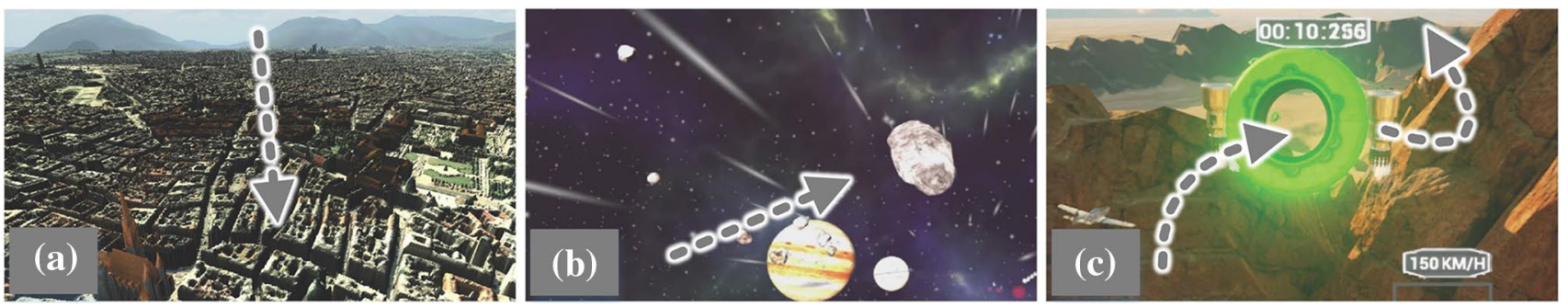

Fig. 7 For the experiment, we employed three types of content with different degrees of freedom and complexity: skydiving (a no flight controller), space flight (b left-right movement), and air race (c full 3D movement)

discusses the questionnaire we formulated for providing the data basis of the hypothesis testing process.

\subsubsection{Test environment}

The essential component of the test environment is the Jumpcube itself. Applied in three real-world situations for 7 days over 1 month (see Sect. 3.3 for details) curious volunteers were invited to perform a jump. After the jump sequence subjects were asked to fill out a one-page questionnaire. Almost 200 subjects provided feedback.

The jump sequence consists of the following steps: (1) jump preparation ending with standing at the rim of the ramp, (2) jumping, (3) horizontal flight, (4) raising and, (5) landing. Mapped on the skydiving content, (1) is pictured as standing in an airplane, (2) as jumping out of the door, (3) as flying through clouds down towards the city of Vienna, (4) as parachute opening, (5) crashing through the roof of TU Wien and landing in front of the rector. The average duration of the jump sequence is $5 \mathrm{~min}$. The total experience with preparation, jumping and releasing takes approximately eleven minutes. Average subjects needed $5 \mathrm{~min}$ to complete the questionnaire.

As it is freely financed, presentation events are of essential importance for the Virtual Jumpcube project. Hence, the evaluation was limited in a number of ways. First, all subjects were real-world customers that were not briefed into the experiment. In consequence, the evaluation had to be short and use general language, thus limiting the complexity and depth of the questions. Furthermore, since all events were semi-public exhibitions, test subjects had the opportunity to watch others jumping which might influence their perception and opinion of the virtual experiences. Finally, the most relevant limitation from the author's perspective is that the evaluation had to be performed directly after the jump since otherwise the subjects would have left the Jumpcube area which would probably have reduced the response rate significantly. Just $5 \mathrm{~min}$ after the jump most subjects are visibly excited which might influence their judgment. Unfortunately, given the nature of the project and the experiment, we were not able to eliminate this potential influence on the results. Nevertheless, at least the influencing excitement can be considered as genuine and originates in the experience itself.

\subsubsection{Employed virtual reality contents}

Figure 7 shows samples from the three employed contents. Before the jump, the subjects were allowed to choose their scenario freely. The three options skydive (a), space travel (b) and air race (c) represent three levels of difficulty. All three experiences follow the above-described jump sequence and are of equal length. It is important to note that in the skydiving application the subject cannot influence the flight direction. In the space flight, the subject can move in one dimension going left and right to catch space trash (old satellites). In the air race, the subject can move in two dimensions to catch flying rings. Flight control in the space application is implemented in a self-developed acceleration sensor attached to the breast plate of the jump suit. For the air race we employ gaze control: the subject flies wherever he or she looks.

Since the purpose of the experiment is hypothesis testing independent of the employed content, all three content variants employ the same olfactory and haptic stimuli with the same parametrization. That is, the same set of smell stimuli is employed by all three contents and the amount of smell as well as the frequency/exposure times are the same in all three applications. This is equally true for the haptic stimuli where the weather system, force simulation and suspension are employed in exactly the same way over all three contents. Finally, though the audiovisual content is semantically different for the three applications, the technical parameters (spatio-temporal resolution, quality of content, quality of service, etc.) are the same for all three. Thus, we provide a situation where the customer/test subject has a free choice of content flavors while limiting the influence on the generality of the experiment to the minimum.

Technically, in all applications, audiovisual content is encoded in a proprietary description format (a simple JSON document) that states what audible/visual/ olfactory/haptic media are employed at which absolute times. These scrips are executed by a game engine (unity for skydiving and 
Table 2 English version of the questionnaire for the present evaluation

(A) Questions on the olfactory stimuli (if possible, tick the most adequate answer)

1. Did you perceive smells in the virtual reality experience?

2. If 1 . yes, were the smells pleasant?

3. If 1 . yes, how intense were the smells?

4. If 1 . yes, did the smells make the virtual reality experience (VR experience) more interesting?

5. If 1. yes, were the smells at the same time as the linked events (e.g., airplane/motor smell)? Yes, mostly

6. If 1 . yes, were the smells adequate for the linked audiovisual events?

Yes, mostly

Positively

Yes

No

7. If 1 . yes, how did you perceive the smell of the airplane/space ship?

Yes, certainly

8. Do you think that smells could generally be an interesting component of VR experiences?

(B) Questions on the haptic stimuli (please judge only those stimuli that were actually present)

1. Was the physical jump exciting for you?

2. Did you notice the humidity of the clouds?

3. Was the flight through the clouds exciting for you?

4. Did you recognize the centrifugal forces in curves?

5. If yes, were the centrifugal forces realistic?

6. Did you recognize the turbulences during the landing?

7. Was the landing process exciting for you?

8. Was the flight through the building exciting for you?

(C) General questions

1. Age of the jumping subject:

2. Sex/gender of the jumping subject:

3. Did you have VR experience before your jump?

4. Was our VR experience exciting for you?

5. Did you forget the outside world during the experience?

Yes, very Rather yes No

Yes

Yes, very Rather yes

Rather yes

No, negative

Too strong Adequate

Too weak

Yes, a bit

No, negative

Sometimes

S, never

Sometimes

Seldom, never

Neutral, not

Negatively

No, not sure

6. Did our VR experience cause nausea?

7. How good is your sense of smell?

(D) Jo be filled out by the operating team

1. Observed jump style:

2. Employed VR content:

Yes

Yes, very Rather yes

Yes

Yes, very

Rather yes

Yes, very

Rather yes

No

No

No

No

No

No

Up to 18 years

19-49 years

No

Female

Male

$50+$ years

Yes, much

Yes, a bit

Other

Yes, very

Yes, a little

No

Yes, mostly

Yes, sometimes No

Yes, strongly

Yes, a little

No

Very good

Good, normal

Not good

Extraordinary Brave

Relaxed

Skydive

Mars

Airrace

Due to the nature of the events (non-scientific, paid Jumpcube participation), the answer sets were reduced to a minimum of expressive choices to minimize completion time

space flight, unreal for the air race), events along the flight path are triggered by collider objects and all controllers are-as described above-addressed through a standardized IP socket. Colliders are positioned early in the content so that observed latencies have no effect on the synchronous provision of all dimensions/stimuli of a multimedia event.

It is important to note that the contents were carefully designed to create a maximum of excitement while not causing motion sickness. This is important for the economic sanity of the project but it makes the evaluation task even harder because nausea is a rare event in the Jumpcube. The positive side of this fact is that it makes the experiment more realistic than a deliberately nausea-triggering lab experiment.

\subsubsection{Questionnaire}

Table 2 depicts the questionnaire employed in the evaluation. It consists of four sections of which the first three are filled out by the participant and the last section by the operating personnel. Section A refers to the olfactory experience, Section B to the haptic stimuli and Section C collects demographic data. The latter are discussed in the next section. Due to the limited amount of time available, questions were formulated as simply as possible and the number of answer categories was limited to the absolute minimum. In consequence, hypothesis testing was technically performed by binomial testing. The details are explained in the next section.

\subsection{Venues and participants}

Three paid events were chosen for the present evaluation of smell and touch in the Virtual Jumpcube:

- European Congress of Radiology 2017 (ESR17): The European Congress of Radiology is an annual confer- 
Table 3 Demographics of venues and participants

\begin{tabular}{llllll}
\hline Venue & $\begin{array}{l}\text { \#Partici- } \\
\text { pants }\end{array}$ & \%Females & $\% 19-49 y$ & $\begin{array}{l}\text { \%Experi- } \\
\text { enced }\end{array}$ & \%Anosmia \\
\hline ESR17 & 84 & 42 & 86 & 36 & 7 \\
NextM & 33 & 64 & 94 & 55 & 3 \\
VCM & 79 & 41 & 61 & 38 & 10 \\
Total & 196 & 45 & 77 & 40 & 8 \\
\hline
\end{tabular}

The column "19-49y" refers to the age group of 19-49 years, "experienced" sums up all participants with at least some virtual reality experience

ence and expo in Vienna with approximately 27,000 participants. It is mostly attended by doctors and graduate students of medicine. Since virtual reality is gaining more and more attention in medicine, and in particular, in radiology, the Virtual Jumpcube was invited 2016 and 2017 as a show program to demonstrate doctors the power of this technology.

- GroupM NextM Conference 2017 (NextM): NextM is an annual conference on future trends in marketing held 2017 in Vienna. Most participants are executives of large national and international companies. Again, the Jumpcube was invited to demonstrate in an entertaining way the potentials of virtual reality as a multimedia show.

- Exhibition Opening at the Vienna City Museum (VCM): The VCM exhibits artifacts on the history of the city. Exhibitions change in average every 6 months. For the new exhibition starting in Spring 2017, the Jumpcube was invited as an interactive showcase and presented for 1 weekend to the paying visitors.

Table 3 lists statistical information on the participants of the evaluation. Altogether 196 subjects participated. At ESR17, 84 subjects filled out the questionnaire after the jump. Of these $42 \%$ were females and $86 \%$ were between 19 and 49 years old. Surprisingly, only $36 \%$ declared previous virtual reality experience though the expo part of the congress showed a significant number of imaging systems that use virtual reality both in 2016 and 2017 . Only $7 \%$ of the participants rated their sense of smell as "bad". The NextM statistics are substantially different. Here, hardly any elderly people participated and almost two thirds of the participants were woman. An outstanding 55\% of the participants had already had virtual reality experience and a surprisingly small number of just 3\% declared (partial) anosmia. If this were true it would be significantly below the estimated average of the population of 10-15\% [12]. Eventually, the VCM had a more diverse audience. Only $61 \%$ were neither youngsters nor elderly citizens, $38 \%$ had virtual reality experience and $10 \%$ admitted a bad sense of smell. In summary, the vast majority of the participants were between 19 and 49 years old, the genders were almost balanced, $40 \%$ had experience with the technology and approximately $92 \%$ declared themselves as able to perceive smells.

\section{Results}

Below, we investigate whether the fundamental assumption that smell and touch increase immersion and excitement while reducing the risk of nausea held during the evaluation. In the first section, we provide descriptive statistics, thereby endeavoring to make the comprehensive response data more tangible. The second section describes the evaluation of the hypotheses defined above. Finally, we discuss the results in the third sections.

\subsection{General findings}

Figure 8 illustrates the statistics over all events, genders and age groups. The indicators $X n$ refer to the questions $n$ of sections $X$ of the questionnaire shown in Table 2 . Answers are encoded as cardinal numbers $0-3$ where 0 signifies "no answer", 1 the first option, and so on.

The statistics on participant behavior show that most jumpers prefer a conservative jump style at their first jump, which is not surprising. Of the $10 \%$ daring jumpers, $65 \%$ were male and only $5 \%$ were youngsters compared to almost $8 \%$ in the total sample: young subjects were more risk-averse than elder subjects. It appears noteworthy that $65 \%$ of the daring jumpers had prior virtual reality experience compared to only $40 \%$ in the entire sample. That seems to imply that users with virtual reality experience trusted the installation more than users without that knowledge - though the risk of injury is not related to the virtual reality part of the installation. Concerning age vs. experience, $47 \%$ of the young participants had prior experience compared to $40 \%$ in the adults and $33 \%$ in the elder subjects; $32 \%$ of the female participants had virtual reality experience compared to $46 \%$ of the males.

Content statistics: the preferred content was the skydiving application (58\% compared to 27 and $15 \%$ for space flight and air race, respectively). Of the female participants, even $63 \%$ of the subjects chose the skydiving application. Only $6 \%$ of the skydivers showed a daring jump, whereas $26 \%$ of the air racers performed an extraordinary dive. The correlation is understandable since the air race was presented to the clients as potentially causing nausea, and therefore, mostly chosen by risk-taking individuals. Over all contents, $65 \%$ of the participants reported very high immersion and $79 \%$ very high excitement. The values for the skydivers lie exactly in the global average. Interestingly, though air racers experience even higher levels 
Fig. 8 Evaluation results: skydiving was the preferred application (D1) and most subjects were not very daring jumpers (D2). Genders were fairly equally distributed (C2). The overwhelming majority found the VR experience exciting (C4), forgot the outside world (C5) and experienced no nausea (C6). See Table 2 for the meanings of the categories (e.g., C1). $R=0$ means that no answer was given, $R=n$ means that the $n$-th answer was selected by the subject. All quantities are given in per cent

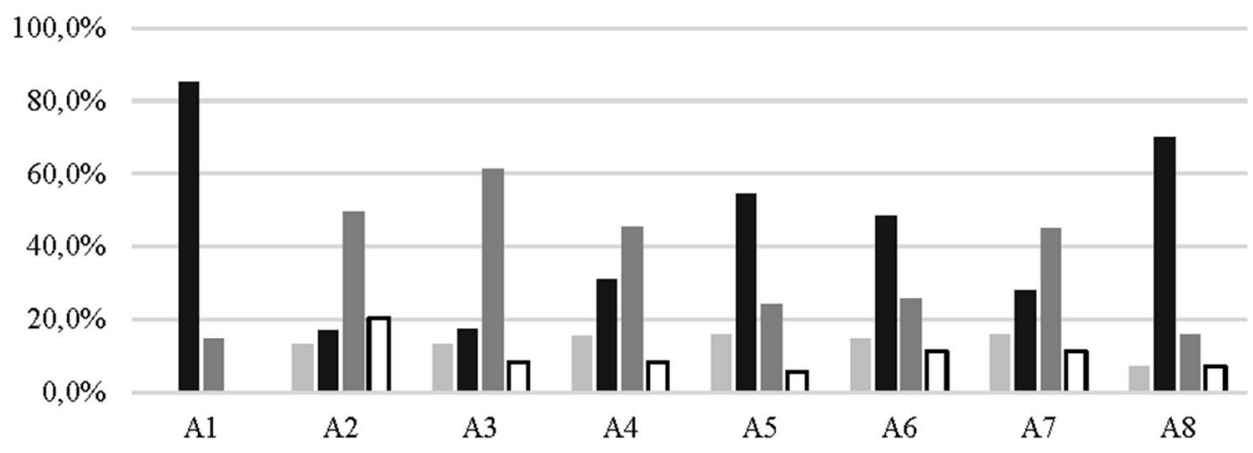

$100,0 \%$
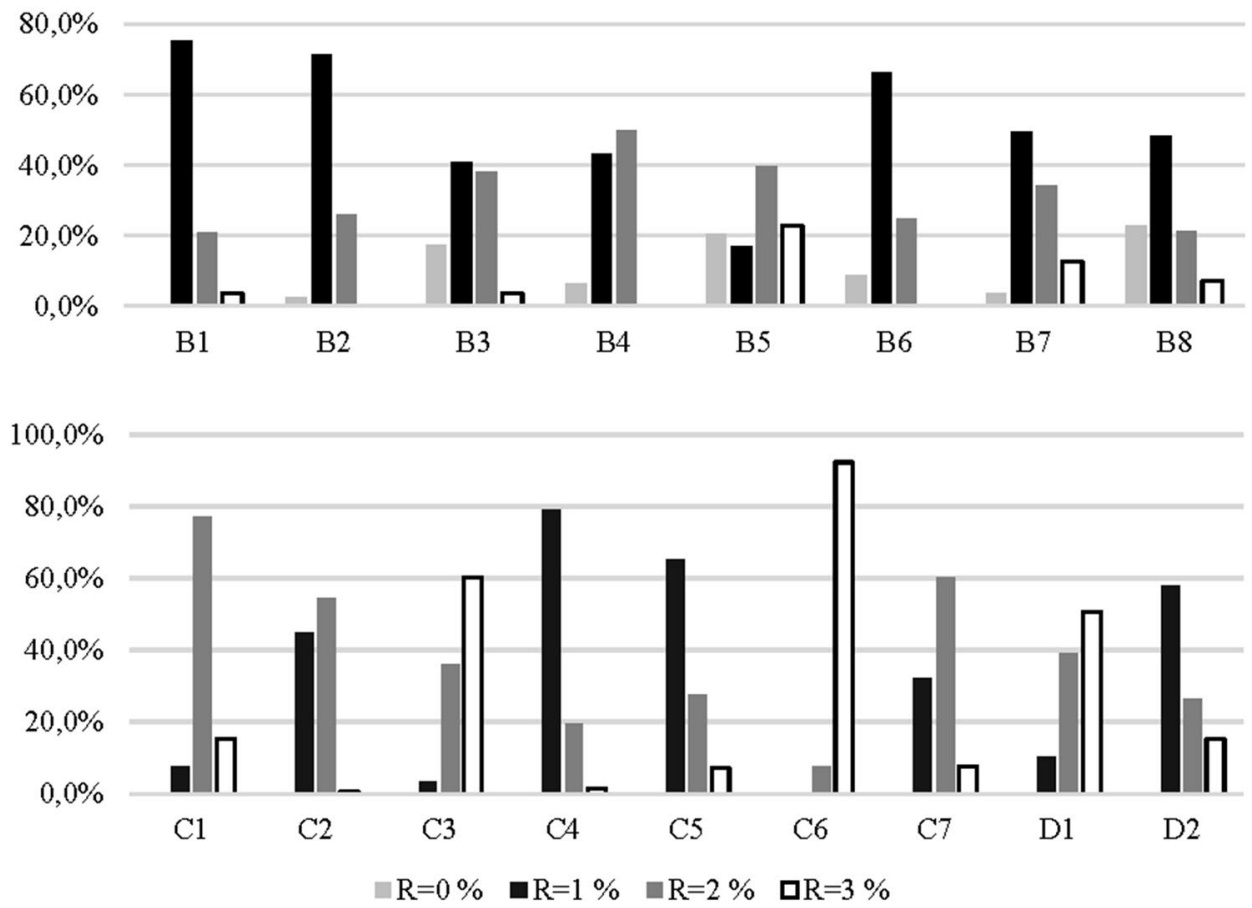

of excitement (83\%) their chance of very high immersion is slightly lower than the average (63\%). It might be concluded that excitement is not just a function of immersion but might also be triggered without perfect virtuality. Finally, the average risk of experiencing nausea was only $8 \%$. For skydiving, it was even lower $(5 \%)$ and for the air race it was $17 \%$. Hence, for the evaluation of the hypotheses on motion sickness, we mostly depend on the 30 subjects that chose the air race content.

Next, we investigated the general feedback on the Vragrancer. $85 \%$ of the participants perceived the smells present in the multimedia content. The non-perceiving $15 \%$ are twice the number of subjects that declared (partial) anosmia. After filling out the questionnaire some participants asked whether smells had actually been present and were surprised to hear that this had permanently been the case. Independent of the perception of smells, $86 \%$ of the participants replied that smells enrich the virtual reality. Of those subjects that perceived smells even $93 \%$ consider olfactory stimuli an interesting enrichment. This positive result appears to justify our approach to use smells only for selected events, thus causing a higher perceptual effect than through constant application.

Of the smell-perceiving participants, $32 \%$ considered the smell of bypassing flying objects (a synthetic combustion engine smell used for airplanes and space ships) as positive-though it is fair to say that objectively this smell is not pleasant. Even so, the combustion scent seems to emphasize the multimedia event "flying object" which causes a subjective rating of the smell that differs significantly from the objective judgment. In total, $63 \%$ of the smell-perceiving subjects found the scents very well synchronized with their multimedia events. Only $7 \%$ could not match them. $55 \%$ of the subjects found the smells adequate 
Table 4 Operationalization: data sets employed for hypothesis testing

\begin{tabular}{|c|c|c|c|c|}
\hline$H_{0}$ & $p$ numerator & $p$ denominator & $p_{0}$ numerator & Rule \\
\hline H.O1 & $\mathrm{A} 5=$ yes $\& \mathrm{C} 5=$ yes & $\mathrm{A} 5=$ yes & $\mathrm{C} 5=$ yes & $p \geq p_{0}$ \\
\hline H.O2 & $\mathrm{A} 2=$ yes $\& \mathrm{~A} 3=\mathrm{ok}$ & $\mathrm{A} 3=\mathrm{ok}$ & $\mathrm{A} 2=$ yes & $p \geq p_{0}$ \\
\hline H.O3 & $\mathrm{A} 1=$ yes $\& \mathrm{C} 6=$ yes & $\mathrm{A} 1=$ yes & C6 = yes & $p \leq p_{0}$ \\
\hline H.O4 & $\begin{array}{l}\mathrm{A} 1=\text { yes } \& \mathrm{C} 4= \\
\text { very }\end{array}$ & $\mathrm{A} 1=\mathrm{yes}$ & $\mathrm{C} 4=$ very & $p \geq p_{0}$ \\
\hline H.H1 & $\begin{array}{l}\mathrm{B} 1=\text { yes } \& \mathrm{C} 1= \\
50+\end{array}$ & $\mathrm{C} 1=50+$ & $\mathrm{B} 1=$ yes & $p=p_{0}$ \\
\hline H.H2 & $\mathrm{B} 4=$ yes $\& \mathrm{C} 6=$ yes & B4 = yes & C6 = yes & $p \leq p_{0}$ \\
\hline H.H3 & $\begin{array}{l}\text { C5 = yes \& D2 = } \\
\text { Sky }\end{array}$ & $\mathrm{D} 2=\mathrm{Sky}$ & $\mathrm{C} 5=$ yes & $p=p_{0}$ \\
\hline H.H4 & $\mathrm{B} 2=$ yes $\& \mathrm{~B} 3=$ yes & $\mathrm{B} 2=$ yes & B3 = yes & $p \geq p_{0}$ \\
\hline H.G1 & $\begin{array}{l}\mathrm{A} 1=\text { yes } \& \mathrm{~B} 2=\text { yes } \\
\& \mathrm{~B} 4=\text { yes \& C5 } \\
=\text { yes }\end{array}$ & $\begin{array}{l}\mathrm{A} 1=\text { yes \& B2 } \\
\quad=\text { yes \& B4 } \\
=\text { yes }\end{array}$ & $\mathrm{C} 5=$ yes & $p \geq p_{0}$ \\
\hline H.G2 & $\mathrm{C} 4=$ yes $\& \mathrm{C} 5=$ yes & C5 = yes & $\mathrm{C} 4=$ yes & $p \geq p_{0}$ \\
\hline H.G3 & $\begin{array}{c}\mathrm{A} 1=\text { yes } \& \mathrm{~B} 2=\text { yes } \\
\& \mathrm{~B} 4=\text { yes \& } \mathrm{C} 3 \\
=\text { yes \& } \mathrm{C} 4=\text { yes }\end{array}$ & $\begin{array}{l}\mathrm{A} 1=\text { yes \& B2 } \\
\quad=\text { yes \& B4 } \\
=\text { yes \& C3 } \\
=\text { yes }\end{array}$ & $\mathrm{C} 4=$ yes & $p \geq p_{0}$ \\
\hline
\end{tabular}

for their multimedia events which shows that synthetic smells in combination with other channels are able to form a complex, rich-in-detail media object.

The last group of descriptive statistics concerns the touch stimuli employed in the Jumpcube. For example, together with visual stimuli the suspension system was employed to simulate turbulences and crashing through the window of a building. Whereas $96 \%$ of the participants found the actual jumping exciting, $91 \%$ found the landing (with turbulences) exciting. Of those participants who had a crash through the window in their content, again $91 \%$ found it exciting. There appears to be no gender aspect in experienced excitement: though in situ it was expressed very differently by members of the two genders, the feedback is about the same for both.

An interesting result concerns clouds and spray-only present in the skydiving application. Of those participants that perceived spray (94\%) $97 \%$ found the flight through the spray-enriched clouds exciting. Of the others, only $43 \%$ found the clouds exciting and $29 \%$ did not even notice them (compared to just $2 \%$ of the spray-perceiving subjects). These results show clearly how important a simple haptic stimuli can be for raising content awareness. 99\% of the spray-perceiving subjects found their virtual skydive exciting, compared to only $86 \%$ of those who did not perceive spray.

The presented findings are only a small sample from the patterns present in the data. A long sequence of other examples could be given. Still, after drawing a sketch of the results it appears beneficial to move on to the formal analysis of the data.
Table 5 Counts, ratios and results of hypothesis testing

\begin{tabular}{lllll}
\hline$H_{0}$ & $p$ & $p_{0}$ & Test value & $\begin{array}{l}\text { Significant } \\
\text { at } \alpha=0.05 \\
?\end{array}$ \\
\hline H.O1 & $102 / 107=0.953$ & $182 / 196=0.929$ & 0.887 & No \\
H.O2 & $92 / 120=0.767$ & $130 / 196=0.663$ & 0.995 & Yes \\
H.O3 & $13 / 167=0.078$ & $15 / 196=0.077$ & 0.516 & No \\
H.O4 & $132 / 167=0.790$ & $155 / 196=791$ & 0.526 & No \\
H.H1 & $28 / 30=0.933$ & $189 / 196=0.959$ & 0.350 & No \\
H.H2 & $5 / 85=0.059$ & $15 / 196=0.077$ & 0.788 & No \\
H.H3 & $105 / 114=0.921$ & $182 / 196=0.929$ & 0.715 & No \\
H.H4 & $132 / 140=0.943$ & $155 / 196=0.791$ & 1.000 & Yes \\
H.G1 & $52 / 55=0.945$ & $182 / 196=0.929$ & 0.762 & No \\
H.G2 & $179 / 182=0.984$ & $193 / 196=0.985$ & 0.529 & No \\
H.G3 & $24 / 24=1.000$ & $193 / 196=0.985$ & 1.000 & Yes \\
\hline
\end{tabular}

\subsection{Hypothesis testing}

Due to the nature of the answer categories in the questionnaire, the hypotheses had to be tested with binomial tests. For the hypotheses listed in Table 1, Table 4 lists the subsets that were used to calculate the test variable $p$ and the default value $p_{0}$ of the binomial tests. Again, $X n$ refers to question $n$ of section $X$ of the questionnaire in Table 2. The denominator of $p_{0}$ is not given as it is always the total of the participants $(n=196)$. The "rule" column refers to the testing method: two-sided $\left(p=p_{0}\right)$ or one-sided-depending on the formulation of the hypothesis.

In Table 4, "yes" summarizes the answer categories "yes, very" and "rather yes". One exception is hypothesis H.O4 where only those subjects were considered that found the experience very exciting. All categories not mentioned in the table are unconstrained $(X n=*)$. Furthermore, we did not employ category A4 for the measurement of interestingness, because during the evaluation it became clear that most participants were overtaxed with answering this question objectively.

Table 5 lists the results of the testing process. For $p$ and $p_{0}$, the numbers of hits (set sizes) are given as well as the ratios. The test statistics should be larger than $1-\alpha$ to express significance. Since the binomial test is not able to test on a particular $\alpha$, we base the discussion of the results on the test values.

The semantics of the results are discussed in the next section. Formally, H.H4 and H.G3 are the hypotheses with the highest significance. That is, spray is an important tactile stimulus and experienced subjects perceive smell and touch more strongly. For hypothesis H.G3, however, (as for H.H2 and H.O3) the numbers on which these results are based are rather low. Hence, conclusions can only be drawn cautiously. Most other hypotheses stand on a solid numerical basis. 
Some are expressive: H.H2/3, H.O1/2, H.G1. Hypothesis H.H1 does clearly not hold. The other hypotheses are neither here nor there: proof for their correctness could not be found.

\subsection{Discussion}

Below, we discuss the major results of the evaluation, starting with olfactory stimuli, followed by tactile stimuli and, eventually, general findings. The results of hypothesis testing are augmented with the descriptive statistics discussed in the Sect. 4.1.

\subsubsection{The role of smell in virtual reality}

Now, does the usage of smells in the Virtual Jumpcube increase the levels of immersion and excitement while mitigating potential nausea? Some results support our theory while no results seem to contradict it. The application of few olfactory stimuli carefully synchronized with the other media channels appears to have a positive impact on the quality of perception.

One fact that supports this conclusion with a significance level of $99.5 \%$ is hypothesis H.O2: olfactory stimuli with adequate intensity are rather perceived as pleasant independent of their actual smell. That is, if a smell fits the other dimensions of a multimedia event, participants rated it as adequate - thus making the virtual world more realistic and rich in details which should rather have a positive than a negative effect on the level of immersion.

A striking example for this type of judgment is the perception of the engines of flying objects. Though the smell is clearly unpleasant (it stinks) one third of the participants were willing to rate it as positive. Our explanation is that the smell is considered as augmenting the sensation of the bypassing plane or spaceship, thus contributing to the expressiveness of the virtual world.

Furthermore, the conclusion is directly supported by hypothesis H.O1 which reaches a significance level of $88.7 \%$ : subjects that perceive olfactory stimuli as synchronous with the virtual reality content reach a rather higher level of immersion. Since this result is based on more than one hundred participants, it must be considered a major indicator for the usefulness of smells in virtual reality. We found no indication that smells hinder immersion while often, they contribute significantly to reaching a high level of immersion.

Subjectively, $86 \%$ of the participants replied that smells enrich the virtual world. Of those subjects that perceived smells even 93\% found smells an interesting media channel. There is a limitation in the fact that olfactory stimuli seem to have no particular effect on the levels of excitement in the Virtual Jumpcube: $98 \%$ of the smell-perceiving participants found the experience exciting, which is exactly the same result as for the total test group. Of course, at such a high level of excitement it is probably not realistic to expect a significant improvement from one new media channel. It would, therefore, be interesting to evaluate the Vragrancer system in a less exciting virtual reality application (e.g., a training situation). It appears not implausible to us that at lower base levels, olfactory stimuli might contribute to raising the level of excitement.

Finally, we could not identify a link of smell perception and nausea. This might be due to the low number of cases of (slight) nausea in the Virtual Jumpcube. Of the 15 subjects that declared motion sickness, 13 perceived smells and of these $79 \%$ found them pleasant which is an average very similar to the one of the entire test groups. All of these 13 participants consider smells an interesting media channel and all 13 experienced immersion.

In summary, smells increase the level of immersion in the virtual reality applications of the Jumpcube. They might bring excitement into sober business applications of virtual reality, but there is no indication that smells help to reduce the risk of nausea-which might be due to the mostly unpleasant nature of the smells used in the Jumpcube applications (explosions, smoke, plane engines, etc.)

\subsubsection{The influence of tactile stimuli}

The question whether haptic stimuli cause excitement and reduce nausea can be answered with yes. Only the level of immersion need not necessarily be increased by haptic stimulation.

A first result of the evaluation is that physical jumping is exciting (for $96 \%$ of the test group). Elderly subjects have slightly less fun at jumping but are in average as excited as the others after the jump. That justifies the effort for building the complex suspension system of the Virtual Jumpcube: free jumping is significantly more than just flying through a virtual environment.

This finding is supported by the results for the other applications of the suspension system. Of those participants who had a crash through the façade of a building in their content, $91 \%$ found it exciting. Turbulences were perceived by $66 \%$ of the participants and landing was exciting for $91 \%$ of all test subjects. All of these experiences are made possible by the suspension system.

The tactile killer feature is spray caused by water added to the wind. Hypothesis H.H4 reached a significance of 100\%: spray makes clouds rather more exciting. Spray perceivers found in $97 \%$ of the cases flying through the clouds exciting, whereas only $43 \%$ of those who did not perceive the spray founds the clouds exciting. These results show clearly how important this simple tactile stimuli is. Properly synchronized, it turns a simple visual cue into an impressive multimedia event. After the jump, $99 \%$ of the spray-perceiving 
subjects found their skydive exciting compared to $86 \%$ of the non-perceiving subjects. The strong impact of spray might be explained by its tactile effect on the human skin. Spray causes a sensation similar to brushing. Through the recently discovered $\mathrm{C}$ tactile afferents [15], these sensations seem to go directly into amygdala bypassing the hypothalamus, thus causing a guaranteed emotional reaction in the subject. Whatever the neurological explanation is, it is certain that in this case, haptic stimulation causes a strong improvement in virtual reality perception.

On the other hand, we could not identify a link of immersion and excitement for tactile stimulation. Independent of the level of excitement the level of immersion remains on the same level. Contrary to our expectation, excitement and immersion appear to be dimensions of virtual reality that are not almost perfectly correlated with each other. This question will be investigated in detail in the last part of this section.

Finally, hypothesis H.H2 with a significance level of $78.8 \%$ states that the perception of centrifugal forces will rather reduce the risk of nausea. H.H3 (71.5\%) states that centrifugal forces in curves are experienced as realistic no matter if the content is realistic or not. Hence, we have a strong indication that the force simulation system has an effect on motion sickness. It appears to contribute to avoiding nausea. This is the case in realistic scenarios (skydive) but as well in fantastic ones such as the space flight and the air race. Hence, it appears reasonable to include force simulation into advanced virtual reality applications.

\subsubsection{The influence of perception and experience on immersion}

We assume that smell and touch support high levels of immersion if those media channels are in sync with the audiovisual virtual reality content. One fact that supports this idea is hypothesis H.G1 with test statistics of $76.2 \%$ : subjects that perceive olfactory and haptic stimuli more often reach a high level of immersion. That is, the ability to perceive these media channels will per se have a positive effect on the level of immersion.

Second, hypothesis H.G3 with $100 \%$ significance states that subjects with virtual reality experience react stronger on olfactory and haptic stimuli than subjects without experience. We conclude that the non-standard media channels might in particular be means to raise immersion and excitement for the experienced users that are no longer overwhelmed by audiovisual media cues. This finding appears to be of a general nature: in the test data we could not identify a gender or age bias.

Also linked to experience might be the trust in virtual reality applications that appear to be risky. Above we pointed out that $65 \%$ of the bravest jumpers hat prior virtual reality experience. Interestingly, $95 \%$ of these subjects experienced immersion compared to a global average of $92.6 \%$ - a slight improvement. Despite that only $95 \%$ of them are excited while $99 \%$ of the non-daring jumpers are. This fact is another illustration for the finding that immersion and excitement are not necessarily linked. In summary, experience could be a key factor in the development and testing of virtual reality applications. If experience influences the behavior and judgment of subjects, it has to be taken into account in virtual reality user studies.

In summary, it appears safe to state that immersion is not necessarily excitement. Overall, $65 \%$ of the participants reported very high immersion but $79 \%$ reported very high excitement, whereas air racers who experience higher levels of excitement (83\%) have slightly lower levels of immersion $(63 \%)$. These results may be explained by the physical activity required from the participant in the air race. It could be that the haptic feedback from physical exercise might hinder reaching a high level of immersion. This explanation appears to be supported by the findings on tactile stimuli above. For a definite answer though, a more detailed investigation would be required.

\section{Conclusions and future work}

Adding smell and touch to the audiovisual stimuli makes clear that virtual reality is a multimedia problem. Appropriateness and synchronization of the stimulating media channels have a key influence on the degree of immersion that can be reached as well as on the level of excitement-and may reduce the risk of motion sickness. In the present study, we have investigated the Virtual Jumpcube as such a rich media system. Results are that indeed, multimodality potentially increases excitement and that olfactory stimuli increase immersion, whereas force simulation as one particular type of haptic stimulus reduces the risk of nausea. Spray as a very simple tactile stimulus has a strong effect on immersion and excitement. Both media channels, smell and touch, appear to be particularly effective since they are uncommon to most virtual reality users and their neural processing has a tendency to affect directly the emotional centers of the brain such as the amygdala [12].

For the future, it would be beneficial if the game engines employed for virtual reality application programming would support multimedia synchronization standards such as SMIL [18]. For the present project, we had to define a proprietary synchronization mechanism. Since sophisticated methods have been available for a long time, it would be highly desirable to see them implemented in Unity and Unreal, in particular. 
Our future work in the context of this experiment will go into a number of different directions.

1. First, we will implement and test more tactile stimuli. In particular, we will investigate vibration patterns for the stimulation of hands and hips. First experiments on water resistance and shockwaves of bypassing flying objects proved very promising. Vibration patterns could be a generalization of the "spray effect" described in this paper: the simulation of the sensation of brushing the skin that causes $\mathrm{C}$ tactile afferents to fire and raise emotions.

2. Furthermore, we will augment the Jumpcube experience by a taste stimulus. The idea is to provide clients on the ramp before the jump with a candy that has a taste somehow linked to the forthcoming experience (e.g., representing the "freshness" of space or the "fruitiness" of a landscape). Quantitative evaluation will show whether not taste stimulation has an effect on immersion, excitement and motion sickness.

3. We are currently experimenting with biosignal feedback for the early recognition of nausea. If possible, the virtual reality content could be adapted in real time, for example, by reducing the speed, removing curves, adding refreshing smells, etc. First experiments have shown that at least in female subjects a combination of heart rate change rate, blood pressure and oxygen saturation appears to be a relatively stable indicator of emerging nausea. However, for a robust solution significantly more research is required.

4. If we should succeed in acquiring the necessary funds, we will perform further experiments with paid participants and smell/touch stimuli in the Jumpcube then distinguishing between a test group and a comparison group, adding/removing certain stimuli and detailed evaluation of the effects.

In summary, we believe that smell and touch are interesting extensions of semi-static virtual reality installations that can - as the evaluation in the Virtual Jumpcube has shownenhance the experience significantly.

Acknowledgements Open access funding provided by TU Wien (TUW). The author would like to thank all students that were involved in the construction, programming and operating of the Virtual Jumpcube, in particular Dzenan, Florin, Jonas, Juri, Luca and Thomas. Furthermore, this project could not have been realized without the financial support of several Vienna-based companies, in particular, the Waagner Biro steel company, the Doka construction company and the European Society of Radiology. Your help is very much appreciated. Eventually, I would like to thank our over 2000 jumpers so far for their enthusiasm and valuable feedback.

Open Access This article is distributed under the terms of the Creative Commons Attribution 4.0 International License (http://creat ivecommons.org/licenses/by/4.0/), which permits unrestricted use, distribution, and reproduction in any medium, provided you give appropriate credit to the original author(s) and the source, provide a link to the Creative Commons license, and indicate if changes were made.

\section{References}

1. Cakmak, T., Hager, H.: Cyberith virtualizer: A locomotion device for virtual reality. In: SIGGRAPH Emerging Technologies, pp. 6-9 (2014)

2. Chalmers, A., Howard, D., Moir, C.: Real virtuality: a step change from virtual reality. In: Proceedings of the 25 th Spring Conference on Computer Graphics, ACM, pp. 9-16 (2009)

3. Earnshaw, R.A.: Virtual Reality Systems. Academic Press, London (2014)

4. Eidenberger, H., Mossel, A.: Indoor skydiving in immersive virtual reality with embedded storytelling. In: Proceedings of the 21st ACM Symposium on Virtual Reality Software and Technology, ACM, pp. 9-12 (2015)

5. Fels, S., Kinoshita, Y., Chen, T.P.G., Takama, Y., Yohanan, S., Gadd, A., Takahashi, S., Funahashi, K.: Swimming across the pacific: a VR swimming interface. IEEE Comput. Graph. Appl. 25(1), 24-31 (2005)

6. Friedland, G., Jain, R.: Multimedia Computing. Cambridge University Press, Cambridge (2014)

7. Gallace, A., Spence, C.: In Touch with the Future: The Sense of Touch from Cognitive Neuroscience to Virtual Reality. Oxford University Press, Oxford (2014)

8. Gescheider, G.A.: Psychophysics: The Fundamentals. Psychology Press, Abingdon (2013)

9. Eidenberger, H.: Virtual Jumpcube Evaluation Results (2017). http://gut8en.info/docs/vj-questionnaire-q1-17.xlsx. Accessed 17 Aug 2017

10. Icaros GmbH (2017) Icaros Website. http://www.icaros.net/. Accessed 17 Aug 2017

11. Iwata, H.: Artificial reality with force-feedback: development of desktop virtual space with compact master manipulator. ACM SIGGRAPH Comput. Graph. 24(4), 165-170 (1990)

12. Kandel, E.R., Schwartz, J.H., Jessell, T.M., Siegelbaum, S.A., Hudspeth, A.J.: Principles of Neural Science, vol. 4. McGrawHill, New York (2000)

13. Kim, M., Cho, S., Tran, T.Q., Kim, S.P., Kwon, O., Han, J.: Scaled jump in gravity-reduced virtual environments. IEEE Trans. Vis. Comput. Graph. 23(4), 1360-1368 (2017)

14. Li, Z.N., Drew, M.S., Liu, J.: Fundamentals of Multimedia. Springer, Berlin (2014)

15. McGlone, F., Olausson, H., Boyle, J., Jones-Gotman, M., Dancer, C., Guest, S., Essick, G.: Touching and feeling: differences in pleasant touch processing between glabrous and hairy skin in humans. Eur. J. Neurosci. 35(11), 1782-1788 (2012)

16. Ramic-Brkic, B., Chalmers, A.: Virtual smell: authentic smell diffusion in virtual environments. In: Proceedings of the 7th International Conference on Computer Graphics, Virtual Reality, Visualisation and Interaction in Africa, ACM, pp. 45-52 (2010)

17. Rheiner, M.: Birdly an attempt to fly. In: ACM SIGGRAPH 2014 Emerging Technologies, ACM, p. 3 (2014)

18. Rutledge, L., Hardman, L.: Smil: synchronized multimedia integration language. Tonangebend iX Mag. Prof. Inf. Technol. 10, 58-63 (1999)

19. Seidel, R.J., Chatelier, P.R.: Virtual Reality, Trainings Future? Perspectives on Virtual Reality and Related Emerging Technologies, vol. 6. Springer, Berlin (2013)

20. TU Wien (2017) Virtual Jumpcube Website. http://jumpcu.be/. Accessed 17 Apr 2017 